\title{
Combined synchrotron and nonlinear synchrotron-self-Compton cooling of relativistic electrons
}

\author{
R. Schlickeiser ${ }^{1}$, M. Böttcher ${ }^{2}$, and U. Menzler ${ }^{1}$ \\ ${ }^{1}$ Institut für Theoretische Physik, Lehrstuhl IV: Weltraum- und Astrophysik, Ruhr-Universität Bochum, 44780 Bochum, Germany \\ e-mail: rsch@tp4.rub.de \\ 2 Astrophysical Institute, Department of Physics and Astronomy, Clippinger 339, Ohio University, Athens, OH 45701, USA
}

Received 18 January 2010 / Accepted 29 April 2010

\begin{abstract}
The broadband SEDs of blazars exhibit two broad spectral components, which in leptonic emission models are attributed to synchrotron radiation and SSC radiation of relativistic electrons. During high state phases, the high-frequency SSC component often dominates the low-frequency synchrotron component, implying that the inverse Compton SSC losses of electrons are at least equal to or greater than the synchrotron losses of electrons. The linear synchrotron cooling, usually included in radiation models of blazars, then has to be supplemented by the SSC cooling. Here, we present an analytical solution to the kinetic equation of relativistic electrons subject to the combined synchrotron and nonlinear synchrotron self-Compton cooling for monoenergetic injection. We calculate the time-dependent fluxes and time-integrated fluences resulting from monoenergetic electrons cooling via synchrotron and SSC, and suggest this as a model for the broadband SED of Compton-dominated blazars.
\end{abstract}

Key words. radiation mechanisms: non-thermal - BL Lacertae objects: general - gamma rays: galaxies

\section{Introduction}

The new generation of air Cherenkov TeV $\gamma$-ray telescopes such as HESS, MAGIC, and VERITAS (for review see Hinton \& Hofmann 2009), have so far detected 27 AGNs as powerful variable high-energy photon emitters. Apart from the three radio galaxies $\mathrm{M} 87$, Cen $\mathrm{A}$, and 3C 66B, the remaining 24 AGNs all belong to the blazar class that is characterized by rapid time variability at all wavelengths and that often have established superluminal motion components at radio and $\mathrm{mm}$ frequencies. The combination of high observed luminosities with the observed short time variability in blazar flares indicates that the photon emission in blazars originates in relativistic jet knots that are beamed and Doppler-boosted towards the observer (e.g. Schlickeiser 1996).

The broadband continuum spectra of blazars are dominated by nonthermal emission and often consist of two distinct broad components. In leptonic emission models (for review see Böttcher 2007) synchrotron radiation from highly relativistic electrons generates the low-energy component whereas the high-energy component results from Compton interactions of the same relativistic electron population. A prime candidate for the source photons being Compton upscattered are the selfproduced synchrotron photons, which defines the synchrotron self-Compton (SSC) process (e.g., Maraschi et al. 1992; Bloom $\&$ Marscher 1996). Because of the short radiative synchrotron and SSC cooling time scales it is necessary to calculate selfconsistently the time evolution of the energy spectrum of the radiating relativistic electrons when modeling the spectral energy distribution (SED) from these objects.

The SSC emission model has been applied very successfully to represent the SEDs of the high-frequency peaked BL Lac objects PKS 2155-304 (Aharonian et al. 2005), 1ES 1121-232
(Aharonian et al. 2007), and Mrk 421 (Fossati et al. 2008), for which during high state phases the SSC component in the SED dominates over the synchrotron component. The dominance of the $\gamma$-ray (Compton) component in the SED is usually even more pronounced in the lower-peaked blazars, in particular, flat spectrum radio quasars (FSRQs) like PKS 0528+134 (Sambruna et al. 1997) or low-frequency peaked BL Lac objects (LBLs) like BL Lacertae (e.g., Madejski et al. 1998). The recent Fermi survey of bright blazars (Abdo et al. 2010a) also revealed many sources with $\gamma$-ray dominated SEDs including PKS 0227-369, PKS 0347-211, PKS 0454-234, PKS 1454-354, PKS 1502+106, PKS 2325+093, 3C 454.3 (Abdo et al. 2009) and 3C 279 (Abdo et al. 2010b).

In most modelling works reproducing the SEDs of blazars, a single or broken power-law distribution of electrons is assumed to produce both the synchrotron and Compton emission. Comparing the required parameters for modelling FSRQs and LBLs in such a framework, many authors have concluded that these Compton-dominated blazars are more plausibly represented by $\gamma$-ray emission being dominated by Comptonization of external radiation fields, e.g., from the accretion disk or the broad line region (e.g., Madejski et al. 1999; Böttcher \& Bloom 2000). Since this conclusion depends on the assumption of an underlying power-law electron distribution, we here revisit this problem by investigating the radiative signatures of the injection and subsequent, self-consistent synchrotron and SSC cooling of monoenergetic electrons, including the case of SSC-dominated radiative output.

The dominance of the SSC component over the synchrotron component in this case implies that the inverse Compton SSC losses of electrons are at least equal to or greater than the synchrotron losses of electrons, even more when the intergalactic 
attenuation of the $\mathrm{TeV}$ emission from the cosmic infrared background is accounted for. The ratio of the observed SSC to synchrotron photon luminosity from the same population of electrons $n(\gamma)$

$\frac{L_{\mathrm{SSC}}^{*}}{L_{\mathrm{sy}}^{*}}=\frac{\int \mathrm{d} V \int_{1}^{\infty} \mathrm{d} \gamma n(\gamma)\left|\gamma_{\mathrm{SSC}}\right|}{\int \mathrm{d} V \int_{1}^{\infty} \mathrm{d} \gamma n(\gamma)\left|\dot{\gamma_{\mathrm{S}}}\right|}$

directly reflects the ratio of the corresponding loss rates, because of the identical Doppler boosting factors (Dermer \& Schlickeiser 2002) of synchrotron and SSC emission. That a distinct third broad emission component did not appear at high photon energies in blazar SEDs can be interpreted in two ways: (i) if the first-order Compton scattering of synchrotron photons is in the Thomson regime, higher order SSC scattering components then do not operate in the Thomson regime but in the extreme KleinNishina limit with a much reduced scattering cross section; (ii) all SSC components including the first-order one operate in the extreme Klein-Nishina limit so that the maximum photon energy is determined by the initial electron energy modulo beaming. Throughout this work we only consider the first case.

In the case of the dominance of the first-order SSC component over the synchrotron component, Schlickeiser (2009hereafter referred to as paper $S$ ) pointed out that the linear synchrotron cooling rate, usually included in radiation models of blazars, then has to be supplemented by the nonlinear SSC cooling rate. In the Thomson limit (hereafter referred to as SST-losses) the SST energy loss rate of a single electron

$|\dot{\gamma}|_{\mathrm{SST}} \simeq A_{0} \gamma^{2} \int_{0}^{\infty} \mathrm{d} \tilde{\gamma} \tilde{\gamma}^{2} n(\tilde{\gamma}, t), \quad A_{0}=\frac{3 \sigma_{\mathrm{T}} c_{1} P_{0} R \epsilon_{0}^{2}}{m c^{2}}$

depends on the energy integral of the actual electron spectrum $n(\gamma, t)$, reflecting that the energy integral determines the number density of the target synchrotron photons in the source. The dependence on the energy integral is a collective effect completely different from the linear synchrotron energy loss rate of a single electron in a constant magnetic field

$|\dot{\gamma}|_{\mathrm{S}}=D_{0} \gamma^{2}, \quad D_{0}=\frac{4}{3} \frac{c \sigma_{\mathrm{T}}}{m c^{2}} U_{B}=1.29 \times 10^{-9} b^{2} \mathrm{~s}^{-1}$,

which is solely determined by the magnetic field strength $B=b$ Gauss and the electron Lorentz factor $\gamma$. The notation in Eqs. (2) and (3) is the same as in paper S: $P_{0}=3.2 \times 10^{12} \mathrm{eV}^{-1} \mathrm{~s}^{-1}$, $\epsilon_{0}=1.16 \times 10^{-8} b \mathrm{eV}, R$ is the radius of the spherical source, $c$ denotes the speed of light, $\sigma_{\mathrm{T}}=6.65 \times 10^{-25} \mathrm{~cm}^{2}$ is the Thomson cross section, and $c_{1}=0.684$.

The competition between the instantaneous injection of ultrarelativistic electrons $\left(\gamma_{0} \gg 1\right)$ at the rate $Q(\gamma, t)=q_{0} \delta(\gamma-$ $\left.\gamma_{0}\right) \delta(t)$ at time $t=0$ and the electron radiative losses $|\dot{\gamma}|$ is described by the time-dependent kinetic equation for the volumeaveraged relativistic electron population inside the radiating source (Kardashev 1962):

$\frac{\partial n(\gamma, t)}{\partial t}-\frac{\partial}{\partial \gamma}[|\dot{\gamma}| n(\gamma, t)]=q_{0} \delta\left(\gamma-\gamma_{0}\right) \delta(t)$,

where $n(\gamma, t)$ denotes the volume-averaged differential number density. S solved this kinetic equation for the two cases, where the radiative loss rate is given either by the linear synchrotron loss rate (3) or the nonlinear SST loss rate (2), for the illustrative case of instantaneous injection of monoenergetic particles. From the respective relativistic electron number densities he then calculated time-dependent optically thin synchrotron and SSC intensities, and time-integrated total synchrotron and SSC fluence distributions, which can be compared with the observed SEDs of blazars. For the illustration case of instantaneous injection of monoenergetic particles, he found significant differences for both (i) the optically thin synchrotron and SSC radiation intensities and (ii) fluence-energy spectra in the two different cooling cases.

It is purpose of the present analysis to solve the electron kinetic equation for the more realistic case where the radiative loss rate is the sum of the linear synchrotron and nonlinear SST cooling rate, i.e.,

$|\dot{\gamma}|=|\dot{\gamma}|_{\mathrm{S}}+|\dot{\gamma}|_{\mathrm{SST}}=\gamma^{2}\left[D_{0}+A_{0} \int_{0}^{\infty} \mathrm{d} \tilde{\gamma} \tilde{\gamma}^{2} n(\tilde{\gamma}, t)\right]$.

In Sect. 2 we calculate the analytic solution of the electron kinetic equation under the combined synchrotron and SST losses (5) for the case of instantaneous injection of monoenergetic particles. The analytic solution is then used in Sects. 3 and 4 to calculate the optically thin synchrotron radiation intensity and total fluence distribution, respectively. In Sect. 5 we demonstrate that our analytical results agree well with synchrotron intensity and fluence resulting from the numerical radiation code of Böttcher et al. (1997) for this case. This code also provides the SSC intensity and fluence distributions.

In our analytical analysis we only take the synchrotron photons into account as seed photons for electron cooling, and neglect any higher order SSC components. In contrast, the numerical radiation code of Böttcher et al. (1997) consistently accounts for all SSC radiation fields. The comparison of the analytical and numerical synchrotron radiation fluence distributions calculated below will therefore test the validity of the assumptions made in the analytical calculations.

Moreover, the nonlinear SSC electron loss rate (2) is approximated to operate in the Thomson limit. According to $\mathrm{S}$, this limits the initial electron Lorentz factor to $\gamma_{0}<1.94 \times 10^{4} b^{-1 / 3}$, putting an upper limit on the maximum SSC photon energy (modulo beaming) of $\epsilon_{\mathrm{SSC} \text {, } \max }=4 \epsilon_{0} \gamma_{0}^{4}<6.6 b^{-1 / 3} \mathrm{GeV}$. Our study therefore applies more to blazars observed in the Fermi survey, whose SEDs peak at sub-GeV energies, and not to $\mathrm{TeV}$ blazars. Throughout this work we therefore consider emission regions that have all of the first-order SSC emission initially in the Thomson regime. At later times after significant electron cooling, the Thomson approximation is fulfilled even better.

\section{Solution of electron kinetic equation for combined synchrotron and SST cooling}

For combined synchrotron and SST cooling (5) the kinetic equation of the electrons (4) reads

$$
\begin{gathered}
\frac{\partial n(\gamma, t)}{\partial t}-\frac{\partial}{\partial \gamma}\left[\gamma^{2} n(\gamma, t)\left(D_{0}+A_{0} \int_{0}^{\infty} \mathrm{d} \tilde{\gamma} \tilde{\gamma}^{2} n(\tilde{\gamma}, t)\right)\right]= \\
q_{0} \delta\left(\gamma-\gamma_{0}\right) \delta(t) .
\end{gathered}
$$

With the substitution $y=A_{0} t$ and $S(\gamma, t)=\gamma^{2} n(\gamma, t)$, the kinetic Eq. (6) becomes

$\frac{\partial S}{\partial y}-\left[K_{0}+\int_{0}^{\infty} \mathrm{d} \tilde{\gamma} S(\tilde{\gamma}, t)\right] \gamma^{2} \frac{\partial S(\gamma, t)}{\partial \gamma}=q_{0} \gamma^{2} \delta\left(\gamma-\gamma_{0}\right) \delta(y)$

where $K_{0}=D_{0} / A_{0}$. Using $x=1 / \gamma$ as independent variable, we obtain, with $x_{0}=1 / \gamma_{0}$,

$\frac{\partial S}{\partial y}+\frac{\partial S}{\partial x}\left[K_{0}+\int_{0}^{\infty} \mathrm{d} \tilde{x} \tilde{x}^{-2} S(\tilde{x}, y)\right]=q_{0} \delta\left(x-x_{0}\right) \delta(y)$. 
Now we define the implicit time variable $T$ through

$\frac{\mathrm{d} T}{\mathrm{~d} y}=U(y)=K_{0}+\int_{0}^{\infty} \mathrm{d} x x^{-2} S(x, y)$.

Then Eq. (8) becomes

$\frac{\partial S}{\partial T}+\frac{\partial S}{\partial x}=q_{0} \delta\left(x-x_{0}\right) \delta(T)$

which agrees exactly with Eq. (52) of Schlickeiser \& Lerche (2007). According to their Eq. (54) the solution is

$S(x, T)=q_{0} \delta\left(x-T-x_{0}\right)(H[T]-H[T-x])$.

Now we calculate the time variable $T$ explicitly as a function of $y$, using Eq. (11) in Eq. (9) to write

$\frac{\mathrm{d} T}{\mathrm{~d} y}=K_{0}+\int_{0}^{\infty} \mathrm{d} x x^{-2} S(x, y)=$

$K_{0}+q_{0} \int_{0}^{\infty} \mathrm{d} x x^{-2} \delta\left(x-T-x_{0}\right)(H[T]-H[T-x])=$

$K_{0}+q_{0} H[T] H\left[x_{0}\right] \frac{1}{\left(x_{0}+T\right)^{2}}=K_{0}+\frac{q_{0}}{\left(x_{0}+T\right)^{2}}$

for $x_{0}>0$ and $T \geq 0$. With $z(y)=x_{0}+T(y)$, Eq. (12) becomes

$\frac{\mathrm{d} z}{\mathrm{~d} y}=K_{0}+\frac{q_{0}}{z^{2}}=\frac{q_{0}+K_{0} z^{2}}{z^{2}}$

which after separation of variables with the integration constant $C_{1}$ leads to

$K_{0} y+C_{1}=z-\int \frac{\mathrm{d} z}{1+\frac{K_{0} z^{2}}{q_{0}}}=z-\sqrt{\frac{q_{0}}{K_{0}}} \arctan \left(\sqrt{\frac{K_{0}}{q_{0}}} z\right)$,

or

$x_{0}+T(y)-\sqrt{\frac{q_{0}}{K_{0}}} \arctan \left(\sqrt{\frac{K_{0}}{q_{0}}}\left[x_{0}+T(y)\right]\right)=K_{0} y+C_{1}$.

The integration constant $C_{1}$ is fixed by the condition that $T=0$ for $y=0$, yielding

$$
\begin{aligned}
K_{0} y= & T-\sqrt{\frac{q_{0}}{K_{0}}}\left[\arctan \left(\sqrt{\frac{K_{0}}{q_{0}}}\left[x_{0}+T(y)\right]\right)\right. \\
& \left.-\arctan \left(\sqrt{\frac{K_{0}}{q_{0}}} x_{0}\right)\right] .
\end{aligned}
$$

Unfortunately, this dependence $y(T)$ cannot be directly inverted to infer the general dependence $T(y)$. However, an approximate, but reasonably accurate, inversion is possible by using the asymptotic expansions of the arctan-function for small and large arguments compared to unity.

\subsection{Injection parameter}

The argument of the arctan-function is always larger than $\alpha^{-1}=$ $x_{0}\left(K_{0} / q_{0}\right)^{1 / 2}$. Therefore, we consider the two cases $\alpha \gg 1$ and $\alpha \ll 1$, respectively.

The parameter $\alpha$ depends on the energy density of the initially injected relativistic electrons and can be written as

$\alpha=\frac{q_{0}^{1 / 2}}{K_{0}^{1 / 2} x_{0}}=\frac{q_{0}^{1 / 2} \gamma_{0}}{K_{0}^{1 / 2}}=\frac{\gamma_{0}}{\gamma_{B}}$

with the characteristic Lorentz factor

$\gamma_{B}=\frac{K_{0}^{1 / 2}}{q_{0}^{1 / 2}}=\frac{2}{3} \sqrt{\frac{c U_{B}}{c_{1} P_{0} R \epsilon_{0}^{2} q_{0}}}=\frac{106}{R_{15}^{1 / 2} q_{5}^{1 / 2}}$

for standard blazar parameters $q_{0}=10^{5} q_{5} \mathrm{~cm}^{-3}$ and $R=$ $10^{15} R_{15} \mathrm{~cm}$. The $\gamma_{B}$ and $\alpha$ are independent of the magnetic field strength. Note that $\alpha^{2}$ equals the ratio of initial SST to synchrotron losses.

In terms of the total number of instantaneously injected electrons $N=4 \pi R^{3} q_{0} / 3=10^{50} N_{50}$, the characteristic Lorentz factor $\gamma_{B}$ and the injection parameter $\alpha$ scale as

$\gamma_{B}=\frac{217 R_{15}}{N_{50}^{1 / 2}}, \alpha=46 \frac{\gamma_{4} N_{50}^{1 / 2}}{R_{15}}$,

if we scale the electron injection Lorentz factor as $\gamma_{0}=10^{4} \gamma_{4}$. Obviously, the more compact the source, and the more electrons are injected, the smaller the characteristic Lorentz factor $\gamma_{B}$ is. If the injection Lorentz factor $\gamma_{0}$ is higher (smaller) than $\gamma_{B}$, the injection parameter $\alpha$ will be larger (smaller) than unity. For a compact source with a large number of injected relativistic electrons, the injection parameter $\alpha$ is much larger than unity.

In homogenous spherical sources the initial electron injection luminosity is

$$
\begin{aligned}
L_{\mathrm{e}}=4 \pi R^{2} c \gamma_{0} m c^{2} q_{0} & =3 c \gamma_{0} m c^{2} N / R \\
& =7.4 \times 10^{43} \frac{\gamma_{4} N_{50}}{R_{15}} \mathrm{erg} / \mathrm{s},
\end{aligned}
$$

from which one can determine the dimensionless injection compactness of the source

$l_{\mathrm{e}}=\frac{\sigma_{\mathrm{T}} L_{\mathrm{e}}}{3 \pi R m c^{2}}=\frac{\sigma_{\mathrm{T}} c \gamma_{0} N}{\pi R^{2}}=6.35 \times 10^{9} \frac{\gamma_{4} N_{50}}{R_{15}^{2}}$.

The injection parameter (19) scales as $\alpha=5.8 \times 10^{-4} \gamma_{4}^{1 / 2} l_{\mathrm{e}}^{1 / 2}$.

In Appendix A we demonstrate that for low values of the injection parameter $\alpha \ll 1$, corresponding to $\gamma_{0} \ll \gamma_{B}$, the time evolution of the electron distribution function is determined solely by the linear synchrotron losses, and is given by

$$
\begin{aligned}
n\left(\gamma, \gamma_{0}, t\right) & =\frac{q_{0}}{\gamma^{2}} H\left[\gamma_{0}-\gamma\right] \delta\left(\gamma^{-1}-\gamma_{0}^{-1}-D_{0} t\right) \\
& =q_{0} H\left[\gamma_{0}-\gamma\right] \delta\left(\gamma-\frac{\gamma_{0}}{1+D_{0} \gamma_{0} t}\right),
\end{aligned}
$$

which agrees with the standard linear synchrotron cooling solution.

For large injection parameters $\alpha \gg 1$, corresponding to $\gamma_{0} \gg \gamma_{B}$, nonlinear SST losses determine the electron distribution function at early times. As a result, the time evolution of the 
electron distribution function is affected by the nonlinear SST losses only if the injection Lorentz factor $\gamma_{0}$ exceeds the characteristic value $\gamma_{B}$, which is determined by the number of injected electrons and the size of the source. According to Appendix A we obtain for early times

$$
\begin{aligned}
& n_{1}\left(\gamma, \gamma_{0}, t<t_{\mathrm{c}}\right)= \\
& q_{0} H\left[\gamma_{0}-\gamma\right] H\left[t_{\mathrm{c}}-t\right] \delta\left(\gamma-\frac{\gamma_{0}}{\left(1+3 \alpha^{2} \gamma_{0} K_{0} A_{0} t\right)^{1 / 3}}\right) \\
& \quad=q_{0} H\left[\gamma_{0}-\gamma\right] H\left[t_{\mathrm{c}}-t\right] \delta\left(\gamma-\frac{\gamma_{0}}{\left(1+3 q_{0} \gamma_{0}^{3} A_{0} t\right)^{1 / 3}}\right),
\end{aligned}
$$

which agrees with the nonlinear SST solution of S, his Eq. (28). At late times

$n_{2}\left(\gamma, \gamma_{0}, t \geq t_{\mathrm{c}}\right)=$

$q_{0} H\left[\gamma_{B}-\gamma\right] H\left[t-t_{\mathrm{c}}\right] \delta\left(\gamma-\frac{\gamma_{B}}{\frac{1+2 \alpha^{3}}{3 \alpha^{3}}+\gamma_{B} K_{0} A_{0} t}\right)$,

which is a modified linear cooling solution. Both solutions show that at the transition time

$$
\begin{aligned}
t_{\mathrm{c}} & =\frac{\alpha^{3}-1}{3 \alpha^{3} \gamma_{B} D_{0}} \simeq \frac{1}{3 \gamma_{B} D_{0}} \\
& =\frac{2.6 \times 10^{8}}{\gamma_{B} b^{2}} \mathrm{~s}=\frac{1.2 \times 10^{6} N_{50}^{1 / 2}}{R_{15} b^{2}} \mathrm{~s}
\end{aligned}
$$

the electrons have cooled to the characteristic Lorentz factor $\gamma_{B}$.

Summarizing this section, provided electrons are injected with Lorentz factors much higher than $\gamma_{B}$, given in Eq. (18), they initially cool down to the characteristic Lorentz factor $\gamma_{B}$ by nonlinear SST-cooling until time $t_{\mathrm{c}}$. At later times they cool further to lower energies according to the modified cooling solution (24). If the electrons are injected with Lorentz factors much smaller than $\gamma_{B}$, they only undergo linear synchrotron cooling at all energies with no influence from the SST cooling. The characteristic Lorentz factor $\gamma_{B}$ is only determined by the injection conditions, whereas the time scale $t_{\mathrm{c}}$ also depends on the magnetic field strength.

This different cooling behavior for high and low injection energies affects the synchrotron and SSC intensities and fluences, which we investigate in the next sections.

\section{Intrinsic optically thin synchrotron radiation intensities}

In this section we analytically investigate the consequences of the combined synchrotron and SST cooling for the intensity spectra of optically thin synchrotron radiation. We closely follow the earlier analysis in S.

The optically thin synchrotron intensity from relativistic electrons with the volume-averaged differential density $n(\gamma, t)$ is given by

$I(\epsilon, t)=R j_{\mathrm{S}}(\epsilon, t)=\frac{R}{4 \pi} \int_{0}^{\infty} \mathrm{d} \gamma n(\gamma, t) p_{\mathrm{S}}(\epsilon, \gamma)$,

where

$p_{\mathrm{s}}(\epsilon, \gamma)=\frac{P_{0} \epsilon}{\gamma^{2}} C S\left(\frac{2 \epsilon}{3 \epsilon_{0} \gamma^{2}}\right)$

denotes the synchrotron power of a single electron (Crusius \& Schlickeiser 1988) in a large-scale random magnetic field of constant strength $B$.

\subsection{High injection energy}

Inserting the electron density (23) gives at early times $t<t_{\mathrm{c}}$

$I_{1}\left(\epsilon, \tau<\tau_{\mathrm{c}}\right)=\frac{3 R P_{0} q_{0} \epsilon_{0} \epsilon}{8 \pi E_{0}}[1+\tau]^{2 / 3} \operatorname{CS}\left(\frac{\epsilon[1+\tau]^{2 / 3}}{E_{0}}\right)$,

where we have introduced the initial characteristic synchrotron photon energy

$E_{0}=\frac{3}{2} \epsilon_{0} \gamma_{0}^{2}=1.74 b \gamma_{4}^{2} \mathrm{eV}$

and the dimensionless time scale

$\tau=3 A_{0} q_{0} \gamma_{0}^{3} t=3 \alpha^{2} D_{0} \gamma_{0} t=3 \alpha^{2} t / t_{\mathrm{s}}$,

with the linear synchrotron cooling time

$t_{\mathrm{s}}=\frac{7.75 \times 10^{4}}{b^{2} \gamma_{4}} \mathrm{~s}$.

Then

$\tau_{\mathrm{c}}=3 A_{0} q_{0} \gamma_{0}^{3} t_{\mathrm{c}}=\frac{q_{0} \gamma_{0}^{3}}{\gamma_{B} K_{0}} \frac{\alpha^{3}-1}{\alpha^{3}}=\alpha^{3}-1$.

Likewise, inserting the late electron density (24) gives

$$
\begin{aligned}
I_{2}\left(\epsilon, \tau \geq \tau_{\mathrm{c}}\right)= & \frac{R P_{0} q_{0} \epsilon}{4 \pi} \int_{0}^{\gamma_{B}} \mathrm{~d} \gamma \gamma^{-2} \delta\left(\gamma-\frac{3 \alpha^{3} \gamma_{B}}{1+2 \alpha^{3}+\tau}\right) \\
& \times C S\left(\frac{2 \epsilon}{3 \epsilon_{0} \gamma^{2}}\right) \\
= & \frac{R P_{0} q_{0} \epsilon_{0} \epsilon}{24 \pi \alpha^{4} E_{0}}\left(1+2 \alpha^{3}+\tau\right)^{2} \\
& \times C S\left(\frac{\epsilon}{9 \alpha^{4} E_{0}}\left[1+2 \alpha^{3}+\tau\right]^{2}\right)
\end{aligned}
$$

in terms of the same dimensionless time (29).

The function $C S(x)$ is approximated well by (Crusius \& Schlickeiser 1988)

$C S(x)=a_{0} x^{-2 / 3} \mathrm{e}^{-x}$

with $a_{0}=1.151275$ yielding

$$
\begin{array}{r}
I_{1}\left(\epsilon, \tau<\tau_{\mathrm{c}}\right)=\frac{3 a_{0}}{8 \pi} R P_{0} q_{0} \epsilon_{0}\left(\epsilon / E_{0}\right)^{1 / 3}[1+\tau]^{2 / 9} \\
\times \exp \left(-\frac{\epsilon}{E_{0}}[1+\tau]^{2 / 3}\right) \\
\propto\left(\epsilon / \epsilon_{0}\right)^{1 / 3}[1+\tau]^{2 / 9} \exp \left(-\frac{\epsilon}{\epsilon_{1}(\tau)}\right)
\end{array}
$$

and

$$
\begin{aligned}
I_{2}\left(\epsilon, \tau \geq \tau_{\mathrm{c}}\right)= & \frac{3^{1 / 3} a_{0} R P_{0} q_{0} \epsilon_{0}}{8 \pi \alpha^{4 / 3}}\left(\epsilon / E_{0}\right)^{1 / 3}\left[1+2 \alpha^{3}+\tau\right]^{2 / 3} \\
& \times \exp \left(-\frac{\epsilon}{9 \alpha^{4} E_{0}}\left[1+2 \alpha^{3}+\tau\right]^{2}\right) \\
\propto & \left(\epsilon / \epsilon_{0}\right)^{1 / 3}\left[1+2 \alpha^{3}+\tau\right]^{2 / 3} \exp \left(-\frac{\epsilon}{\epsilon_{2}(\tau)}\right),
\end{aligned}
$$




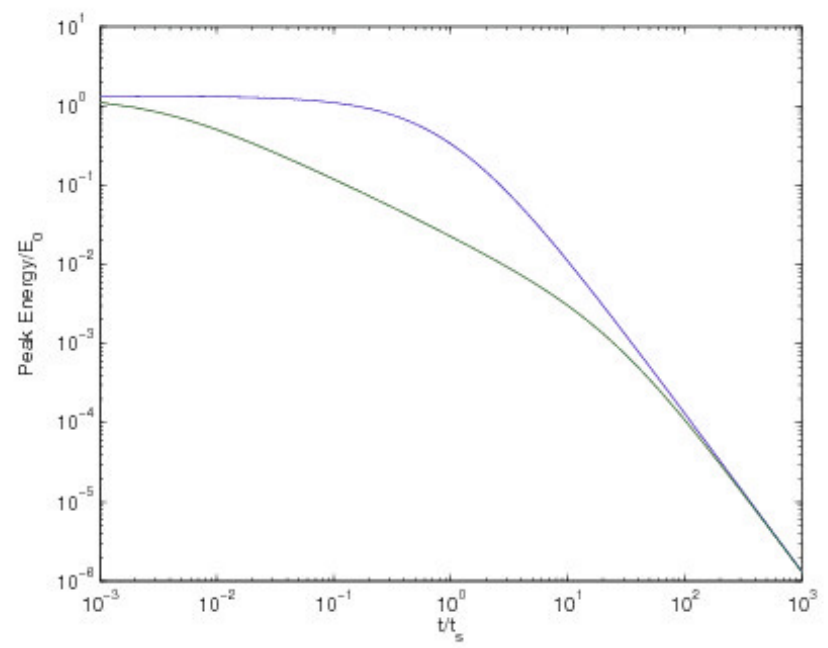

Fig. 1. Time dependence of the peak energy $\epsilon_{\mathrm{p}}(\tau)$ of the synchrotron SED for high ( $\alpha=10$, lower curve) and low ( $\alpha=0.1$, upper curve) values of the injection parameter.

respectively, with the cut-off energies

$\epsilon_{1}\left(\tau \leq \tau_{\mathrm{c}}\right)=E_{0}(1+\tau)^{-2 / 3}$

$\epsilon_{2}\left(\tau \geq \tau_{\mathrm{c}}\right)=9 \alpha^{4} E_{0}\left[1+2 \alpha^{3}+\tau\right]^{-2}$.

With respect to photon energy $\epsilon$, both synchrotron intensities exhibit the same increasing power law with exponential cut-off behavior; however, the cut-off energy differs for early and late times due to the different electron cooling behavior. Note that $\epsilon_{1}\left(\tau_{\mathrm{c}}\right)=\epsilon_{2}\left(\tau_{\mathrm{c}}\right)=E_{0} / \alpha^{2}$.

The cut-off energies (37) determine the time dependence of the peak energy $\epsilon_{\mathrm{p}}(\tau)$ of the synchrotron SED $\epsilon I(\epsilon, \tau)$. At early and late times we obtain

$\epsilon_{\mathrm{p}}\left(\tau<\tau_{\mathrm{c}}\right)=\frac{4 \epsilon_{1}}{3}=\frac{4 E_{0}}{3(1+\tau)^{2 / 3}}=\frac{4 E_{0}}{3\left(1+3 \alpha^{2} \frac{t}{t_{\mathrm{s}}}\right)^{2 / 3}}$

and

$$
\begin{aligned}
\epsilon_{\mathrm{p}}\left(\tau \geq \tau_{\mathrm{c}}\right) & =\frac{4 \epsilon_{1}}{3}=\frac{12 \alpha^{4} E_{0}}{\left[1+2 \alpha^{3}+\tau\right]^{2}} \\
& =\frac{12 \alpha^{4} E_{0}}{\left.\left[1+2 \alpha^{3}+3 \alpha^{2} \frac{t}{t_{\mathrm{s}}}\right)\right]^{2}},
\end{aligned}
$$

respectively, which is illustrated in Fig. 1.

For the high injection case the synchrotron peak energy decreases from its intial maximum value $E_{\mathrm{p}, \max }=\left(4 E_{0} / 3\right)$ proportional to $(1+\tau)^{-2 / 3}$ for early times $\tau<\tau_{\mathrm{c}}=\alpha^{3}-1$ to $E_{\mathrm{p}}=E_{\mathrm{p}, \max } / \alpha^{2}$. At later times $\tau \geq \tau_{\mathrm{c}}$ the peak energy decreases further proportional to $\tau^{-2}$.

\subsection{Low injection energy}

For the low injection energy case $\alpha \ll 1$ we use the electron density (22), which in terms of the normalized time scale (29) reads as

$n\left(\gamma, \gamma_{0}, \tau\right)=q_{0} H\left[\gamma_{0}-\gamma\right] \delta\left(\gamma-\frac{\gamma_{0}}{1+\frac{\tau}{3 \alpha^{2}}}\right)$.
In agreement with $\mathrm{S}$ we obtain for the synchrotron intensity at all times

$$
\begin{aligned}
I(\epsilon, \tau)= & \frac{3 R P_{0} q_{0} \epsilon_{0} \epsilon}{8 \pi E_{0}}\left(1+\frac{\tau}{3 \alpha^{2}}\right)^{2} C S\left(\frac{\epsilon}{E_{0}}\left[1+\frac{\tau}{3 \alpha^{2}}\right]^{2}\right) \\
\simeq & \frac{3 a_{0} R P_{0} q_{0} \epsilon_{0}}{8 \pi}\left(\frac{\epsilon}{E_{0}}\right)^{1 / 3}\left(1+\frac{\tau}{3 \alpha^{2}}\right)^{2 / 3} \\
& \times \exp \left(-\frac{\epsilon}{E_{0}}\left[1+\frac{\tau}{3 \alpha^{2}}\right]^{2}\right)
\end{aligned}
$$

here the synchrotron peak energy

$\epsilon_{\mathrm{p}}=\frac{4 E_{0}}{3\left(1+\frac{\tau}{3 \alpha^{2}}\right)^{2}}=\frac{4 E_{0}}{3\left(1+\frac{t}{t_{\mathrm{s}}}\right)^{2}}$

decreases from its intial maximum value $\left(4 E_{0} / 3\right)$ proportional to $t^{-2}$ for $t>t_{\mathrm{s}}$. The upper curve in Fig. 1 refers to this case. For late times the high and low injection energy cases yield the same $\propto t^{-2}$-decrease of the peak energy.

\subsection{Light-curve peak times}

Equations (38), (39), and (42) also provide the photon energy dependences of the intrinsic light curve peak time or the time of maximum intensity of the synchrotron flare $t_{\max }(\epsilon)$. With Eqs. (31) the peak time is related to $\tau_{\max }(\epsilon)$ as

$t_{\max }(\epsilon)=t_{\mathrm{s}} \frac{\tau_{\max }(\epsilon)}{3 \alpha^{2}}$

with the linear synchrotron cooling time (31).

For the low injection energy case $(\alpha \ll 1)$, we reproduce the well known relation

$t_{\max }(\epsilon, \alpha \ll 1)=t_{\mathrm{s}}\left(\frac{E_{0}}{3 \epsilon}\right)^{1 / 2}-1$

for all energies below $3 E_{0}$.

For the high injection energy case $(\alpha \gg 1)$, we obtain

$t_{\max }(\epsilon, \alpha \gg 1)=t_{\mathrm{s}} \begin{cases}\left(\frac{E_{0}}{3 \epsilon}\right)^{1 / 2}-\frac{2 \alpha^{3}+1}{3 \alpha^{3}} & \text { for } \epsilon \leq \frac{E_{0}}{3 \alpha 2} \\ \frac{1}{3 \alpha^{2}}\left[\left(\frac{E_{0}}{3 \epsilon}\right)^{3 / 2}-1\right] & \text { for } \epsilon \geq \frac{E_{0}}{3 \alpha^{2}},\end{cases}$

indicating a steeper power-law $\left(t_{\max } \propto \epsilon^{-3 / 2}\right)$ at photon energies above $E_{0} / 3 \alpha^{2}$, whereas at lower energies the standard $\left(\propto \epsilon^{-1 / 2}\right)$ dependence results. This is also clearly visible in Fig. 2 where we compare the light curve peak times for low and high injection energy conditions. At high photon energies $\epsilon>E_{0} / 3 \alpha^{2}$ the high injection peak time is a factor $3 \alpha^{2}$ shorter than the low injection peak time. This results from the faster additional SST cooling of relativistic electrons in the high injection case.

\section{Fluences}

To collect enough photons, intensities are often averaged or integrated over long enough time intervals. For rapidly varying photon intensities, this corresponds to fractional fluences given by the time-integrated intensities

$F_{\mathrm{f}}\left(\epsilon, t_{\mathrm{f}}\right)=\int_{0}^{t_{\mathrm{f}}} \mathrm{d} t I(\epsilon, t)=\frac{1}{3 A_{0} q_{0} \gamma_{0}^{3}} \int_{0}^{\tau_{\mathrm{f}}} \mathrm{d} \tau I(\epsilon, \tau)$ 


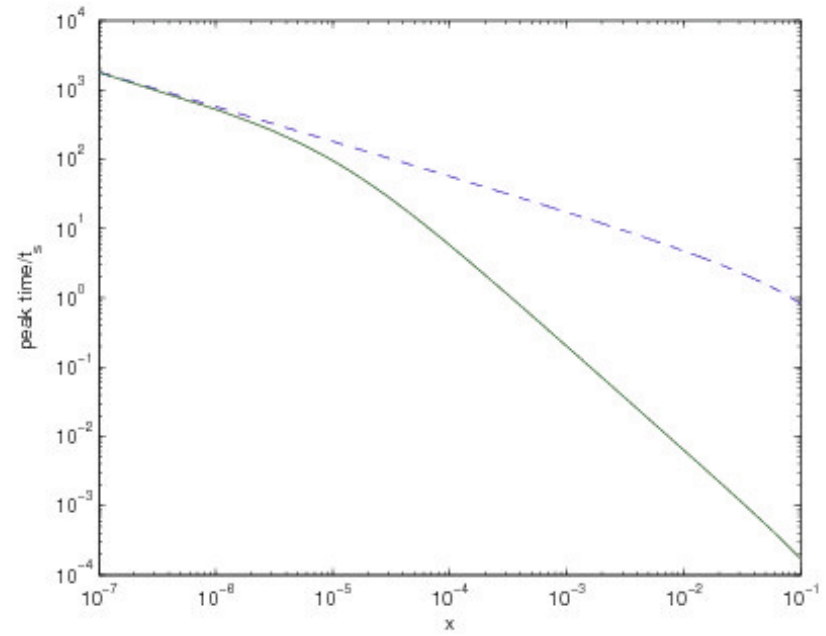

Fig. 2. Photon energy $\left(x=\epsilon / E_{0}\right)$ dependence of the synchrotron light curve peak time for high ( $\alpha=100$, full curve) and low ( $\alpha=0.1$, dashed curve) values of the injection parameter.

with $\tau_{\mathrm{f}}=3 A_{0} q_{0} \gamma_{0}^{3} t_{\mathrm{f}}$. The total fluence spectra result in the limit $t_{\mathrm{f}} \rightarrow \infty$

$$
\begin{aligned}
F(\epsilon)=F_{\mathrm{f}}\left(\epsilon, t_{\mathrm{f}}=\infty\right) & =\int_{0}^{\infty} \mathrm{d} t I(\epsilon, t) \\
& =\frac{1}{3 A_{0} q_{0} \gamma_{0}^{3}} \int_{0}^{\infty} \mathrm{d} \tau I(\epsilon, \tau) .
\end{aligned}
$$

\subsection{Low injection energy}

The synchrotron intensity (41) yields, after obvious substitutions, for the fractional fluence

$$
\begin{aligned}
F_{\mathrm{f}, \mathrm{s}}\left(\epsilon, \tau_{\mathrm{f}}\right)= & \frac{R P_{0} \epsilon}{12 \pi A_{0} \gamma_{0}^{5}} \\
& \times \int_{0}^{\tau_{\mathrm{f}}} \mathrm{d} \tau[1+r \tau]^{2} C S\left(\frac{\epsilon}{E_{0}}[1+r \tau]^{2}\right) \\
= & F_{0 \mathrm{~S}}\left(\frac{E_{0}}{\epsilon}\right)^{1 / 2} \int_{\epsilon / E_{0}}^{\epsilon\left(1+r \tau_{\mathrm{f}}\right)^{2} / E_{0}} \mathrm{~d} x x^{1 / 2} C S(x)
\end{aligned}
$$

with the constant

$F_{0 S}=\frac{R P_{0} E_{0}}{24 \pi r A_{0} \gamma_{0}^{5}}=\frac{R P_{0} \epsilon_{0}}{16 \pi r A_{0} \gamma_{0}^{3}}=\frac{3 \alpha^{2} R P_{0} \epsilon_{0}}{16 \pi A_{0} \gamma_{0}^{3}}$,

and $r=\left(3 \alpha^{2}\right)^{-1}$. The fractional fluence (48) is identical to Eq. $(\mathrm{S}-52)$ in $\mathrm{S}$.

For the total fluence we obtain for low $\left(\epsilon \ll E_{0}\right)$ and high $\left(\epsilon \gg E_{0}\right)$ synchrotron photon energies

$$
F_{\mathrm{S}}(\epsilon) \simeq F_{0 \mathrm{~S}} \begin{cases}c_{0}\left(\frac{E_{0}}{\epsilon}\right)^{1 / 2} & \text { for } \epsilon \ll E_{0} \\ \left(\frac{E_{0}}{\epsilon}\right) \exp \left(-\epsilon / E_{0}\right) & \text { for } \epsilon \gg E_{0},\end{cases}
$$

where (see Appendix B)

$c_{0}=\int_{0}^{\infty} \mathrm{d} s s^{1 / 2} C S(s)=c(1 / 2)=0.95302$.
Likewise, the fractional fractional fluence at high energies is

$$
\begin{aligned}
F_{\mathrm{f}, \mathrm{s}}\left(\epsilon \gg E_{0}, \tau_{\mathrm{f}}\right) \simeq F_{0 \mathrm{~S}}\left(\frac{E_{0}}{\epsilon}\right) \exp \left(-\epsilon / E_{0}\right) \\
\times\left(1-\left(1+r \tau_{\mathrm{f}}\right)^{-1} \exp \left[-\frac{\epsilon}{E_{0}}\left[\left(1+r \tau_{\mathrm{f}}\right)^{2}-1\right]\right]\right) .
\end{aligned}
$$

At small energies $\epsilon \ll E_{0}$ we find for small integration times $t_{\mathrm{f}} \leq\left(E_{0} / \epsilon\right)^{1 / 2} r^{-1}$

$$
\begin{aligned}
F_{\mathrm{f}, \mathrm{s}}\left(\epsilon \ll E_{0}, \tau_{\mathrm{f}} \leq \frac{E_{0}^{1 / 2}}{\epsilon^{1 / 2} r}\right) \simeq \\
\frac{6 F_{0 \mathrm{~S}} a_{0}}{5}\left(\frac{\epsilon}{E_{0}}\right)^{1 / 3}\left[\left(1+r \tau_{\mathrm{f}}\right)^{5 / 3}-1\right],
\end{aligned}
$$

and for late times $t_{\mathrm{f}} \geq\left(E_{0} / \epsilon\right)^{1 / 2} r^{-1}$ the time-independent total fluence spectrum at small energies

$$
F_{\mathrm{f}, \mathrm{s}}\left(\epsilon \ll E_{0}, \tau_{\mathrm{f}} \geq \frac{E_{0}^{1 / 2}}{\epsilon^{1 / 2} r}\right) \simeq F_{0 \mathrm{~S}}\left[\frac{6 a_{0}}{5}+\mathrm{e}^{-1}\right]\left(\frac{\epsilon}{E_{0}}\right)^{-1 / 2}
$$

\subsection{Total fluence for high injection energy}

Here the synchrotron intensities (28) and (33) yield, after obvious substitutions, for the total synchrotron fluence

$$
\begin{aligned}
F_{\mathrm{h}}(\epsilon)= & \frac{1}{3 A_{0} q_{0} \gamma_{0}^{3}}\left[\int_{0}^{\tau_{\mathrm{c}}} \mathrm{d} \tau I_{1}(\epsilon, \tau)+\int_{\tau_{\mathrm{c}}}^{\infty} \mathrm{d} \tau I_{2}(\epsilon, \tau)\right] \\
= & F_{0 \mathrm{~h}}\left(\frac{E_{0}}{\epsilon}\right)^{3 / 2}\left[\int_{\epsilon / E_{0}}^{\epsilon \alpha^{2} / E_{0}} \mathrm{~d} x x^{3 / 2} C S(x)\right. \\
& \left.+\frac{\epsilon \alpha^{2}}{E_{0}} \int_{\epsilon \alpha^{2} / E_{0}}^{\infty} \mathrm{d} x x^{1 / 2} C S(x)\right]
\end{aligned}
$$

with the constant

$$
F_{0 \mathrm{~h}}=\frac{R P_{0} E_{0}}{8 \pi A_{0} \gamma_{0}^{5}}=\frac{3 R P_{0} \epsilon_{0}}{16 \pi A_{0} \gamma_{0}^{3}} .
$$

For high synchrotron photon energies $\epsilon \gg E_{0}$, we approximate

$$
\begin{aligned}
& \int_{\epsilon / E_{0}}^{\epsilon \alpha^{2} / E_{0}} \mathrm{~d} x x x^{3 / 2} C S(x) \simeq \int_{\epsilon / E_{0}}^{\epsilon \alpha^{2} / E_{0}} \mathrm{~d} x x^{1 / 2} \mathrm{e}^{-x} \\
& \simeq\left(\epsilon / E_{0}\right)^{1 / 2} \mathrm{e}^{-\epsilon / E_{0}}-\left(\epsilon \alpha^{2} / E_{0}\right)^{1 / 2} \mathrm{e}^{-\epsilon \alpha^{2} / E_{0}}
\end{aligned}
$$

and

$$
\begin{aligned}
\int_{\epsilon \alpha^{2} / E_{0}}^{\infty} \mathrm{d} x x^{1 / 2} C S(x) & \simeq \int_{\epsilon \alpha^{2} / E_{0}}^{\infty} \mathrm{d} x x^{-1 / 2} \mathrm{e}^{-x} \\
& \simeq\left(\epsilon \alpha^{2} / E_{0}\right)^{-1 / 2} \mathrm{e}^{-\epsilon \alpha^{2} / E_{0}},
\end{aligned}
$$

so that

$F_{\mathrm{h}}\left(\epsilon \gg E_{0}\right) \simeq F_{0 \mathrm{~h}} \frac{E_{0}}{\epsilon} \mathrm{e}^{-\epsilon / E_{0}}$. 
For intermediate synchrotron photon energies $\left(E_{0} / \alpha^{2}\right) \ll \epsilon \ll$ $E_{0}$, we approximate

$$
\begin{array}{rl}
\int_{\epsilon / E_{0}}^{\epsilon \alpha^{2} / E_{0}} \mathrm{~d} & x x^{3 / 2} C S(x) \simeq c_{2}-a_{0} \int_{0}^{\epsilon / E_{0}} \mathrm{~d} x x^{5 / 6} \\
& -\int_{\epsilon \alpha^{2} / E_{0}}^{\infty} \mathrm{d} x x^{1 / 2} \mathrm{e}^{-x} \simeq c_{2}-\frac{6 a_{0}}{11}\left(\epsilon / E_{0}\right)^{11 / 6} \\
& -\left(\epsilon \alpha^{2} / E_{0}\right)^{-1 / 2} \mathrm{e}^{-\epsilon \alpha^{2} / E_{0}} \simeq c_{2}
\end{array}
$$

with the constant

$c_{2}=\int_{0}^{\infty} \mathrm{d} x x^{3 / 2} C S(x)=c(3 / 2)=0.67015$,

and

$\int_{\epsilon \alpha^{2} / E_{0}}^{\infty} \mathrm{d} x x^{1 / 2} C S(x) \simeq\left(\epsilon \alpha^{2} / E_{0}\right)^{-1 / 2} \mathrm{e}^{-\epsilon \alpha^{2} / E_{0}}$,

so that

$F_{\mathrm{h}}\left(\frac{E_{0}}{\alpha^{2}} \ll \epsilon \ll E_{0}\right) \simeq F_{0 \mathrm{~h}} c_{2}\left(\frac{E_{0}}{\epsilon}\right)^{3 / 2}$.

Finally, for low synchrotron photon energies $\epsilon \ll E_{0} / \alpha^{2}$, we approximate

$$
\begin{aligned}
\int_{\epsilon / E_{0}}^{\epsilon \alpha^{2} / E_{0}} \mathrm{~d} x x^{3 / 2} C S(x) & \simeq a_{0} \int_{\epsilon / E_{0}}^{\epsilon \alpha^{2} / E_{0}} \mathrm{~d} x x^{5 / 6} \\
& =\frac{6 a_{0}}{11}\left(\epsilon / E_{0}\right)^{11 / 6}\left[\alpha^{11 / 3}-1\right]
\end{aligned}
$$

and

$$
\int_{\epsilon \alpha^{2} / E_{0}}^{\infty} \mathrm{d} x x^{1 / 2} C S(x) \simeq c_{0}-\frac{6 a_{0}}{5}\left(\epsilon \alpha^{2} / E_{0}\right)^{5 / 6}
$$

with the constant (51). In this case the fluence (55) is

$$
\begin{aligned}
F_{\mathrm{h}}\left(\epsilon \ll \frac{E_{0}}{\alpha^{2}}\right) & \simeq F_{0 \mathrm{~h}} \alpha^{2}\left(\frac{E_{0}}{\epsilon}\right)^{1 / 2}\left[c_{2}-\frac{36 a_{0}}{55}\left(\epsilon \alpha^{2} / E_{0}\right)^{5 / 6}\right] \\
& \simeq F_{0 \mathrm{~h}} \alpha^{2} c_{0}\left(\frac{E_{0}}{\epsilon}\right)^{1 / 2} .
\end{aligned}
$$

For the high injection energy case, the total synchrotron fluence therefore varies as

$$
F_{\mathrm{h}}(\epsilon) \simeq F_{0 \mathrm{~h}} \begin{cases}c_{0} \alpha^{2}\left(\frac{E_{0}}{\epsilon}\right)^{1 / 2} & \text { for } \epsilon \ll E_{0} / \alpha^{2} \\ c_{2}\left(\frac{E_{0}}{\epsilon}\right)^{3 / 2} & \text { for } E_{0} / \alpha^{2} \ll \epsilon \ll E_{0} \\ \left(\frac{E_{0}}{\epsilon}\right) \exp \left(-\epsilon / E_{0}\right) & \text { for } \epsilon \gg E_{0} .\end{cases}
$$

\subsection{Interlude}

At high synchrotron photon energies $\left(\epsilon \gg E_{0}\right)$ the total synchrotron fluences for low and high injection energy exhibit the same exponential cut-off. However, at low energies $\left(\epsilon \ll E_{0}\right)$ we find markedly different power-law behaviors for the two injection cases. In the low injection energy case $(\alpha \ll 1)$, the total synchrotron fluence exhibits the single power-law behavior $\alpha \epsilon^{-1 / 2}$. In the high injection energy case $(\alpha \gg 1)$, the total synchrotron fluence steepens from the power-law $\propto \epsilon^{-1 / 2}$ below $E_{0} / \alpha^{2}$ to the power-law $\propto \epsilon^{-3 / 2}$ above $E_{0} / \alpha^{2}$.

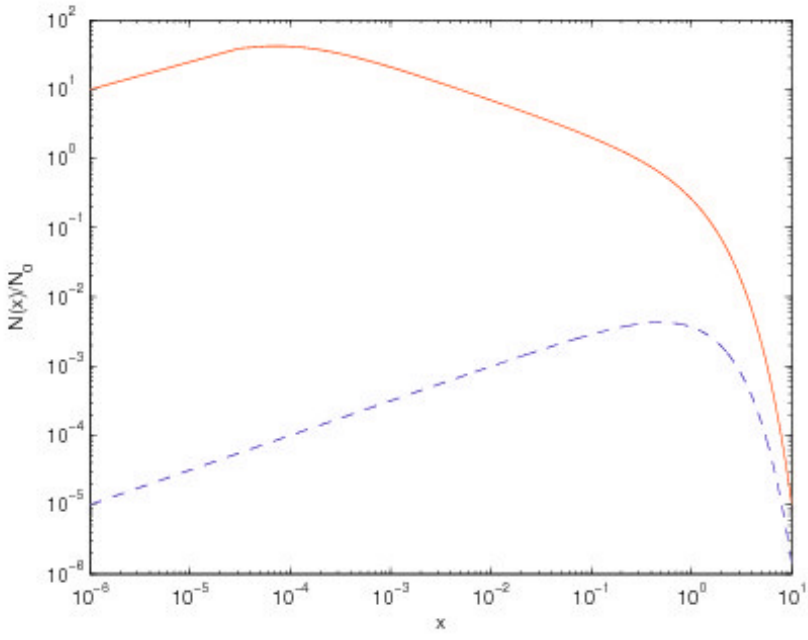

Fig. 3. Total synchrotron fluence SED $N(x)$ for high $\left(\alpha_{\mathrm{h}}=100\right.$, full curve) and small ( $\alpha_{\mathrm{s}}=0.1$, dashed curve) injection conditions calculated for $\gamma_{0}=10^{4}$.

\subsection{Total fluence synchrotron SED}

For the total fluence SED $S(\epsilon)=\epsilon F(\epsilon)$ we then find in the two cases of small (s) and high (h) injection energies

$S_{\mathrm{s}}(\epsilon)=S_{0} \frac{\alpha^{2}}{\gamma_{0}}\left(\frac{\epsilon}{E_{0}}\right)^{1 / 2} \exp \left(-\epsilon / E_{0}\right)$

and

$S_{\mathrm{h}}(\epsilon)=S_{0} \frac{\alpha^{2}}{\gamma_{0}}\left(\frac{\epsilon}{E_{0}}\right)^{1 / 2} \frac{\epsilon_{B}}{\epsilon+\epsilon_{B}} \exp \left(-\epsilon / E_{0}\right)$,

with the constant

$S_{0}=\frac{3 c_{0} m c^{2}}{32 c_{1} \sigma_{\mathrm{T}}}$

and the characteristic break energy

$\epsilon_{B}=\frac{c_{2} E_{0}}{c_{0} \alpha^{2}}=0.703 \frac{E_{0}}{\alpha^{2}}$.

In terms of the normalized synchrotron photon energy $x=\epsilon / E_{0}$ and for the same $\gamma_{0}$, the two total SEDs (68) and (69) are

$N_{\mathrm{s}}(x)=N_{0} \alpha_{\mathrm{s}}^{2} x^{1 / 2} \mathrm{e}^{-x}$

and

$N_{\mathrm{h}}(x)=N_{0} \alpha_{\mathrm{h}}^{2} \frac{x^{1 / 2} x_{B}}{x+x_{B}} \mathrm{e}^{-x}$

with $N_{0}=S_{0} / \gamma_{0}$ and

$x_{B}=\frac{0.703}{\alpha_{\mathrm{h}}^{2}}$.

Figure 3 shows the fluence SEDs $N(x)$ for small $\left(\alpha_{\mathrm{s}}=0.1\right)$ and high $\left(\alpha_{\mathrm{h}}=100\right)$ injection conditions.

In the small injection energy case $N_{\mathrm{s}}$ peaks at $x=0.5$ with the peak value

$N_{\text {s,peak }}=N_{\mathrm{s}}(x=0.5)=\frac{\alpha_{\mathrm{s}}^{2}}{2^{1 / 2}} N_{0} \mathrm{e}^{-1 / 2}$, 
whereas in the high injection case $N_{\mathrm{h}}$ peaks at $x=x_{B}$ with the peak value

$N_{\text {h,peak }}=N_{\mathrm{h}}\left(x=x_{B}\right)=\frac{\alpha_{\mathrm{h}}^{2} x_{B}^{1 / 2}}{2} N_{0} \mathrm{e}^{-x_{B}}$.

The ratio of peak values is given by

$\mathcal{R}=\frac{N_{\mathrm{h}, \text { peak }}}{N_{\mathrm{s} \text {,peak }}}=\frac{\alpha_{\mathrm{h}}^{2} x_{B}^{1 / 2}}{2^{1 / 2} \alpha_{\mathrm{s}}^{2}} \mathrm{e}^{\frac{1}{2}-x_{B}}=$

$0.593 \frac{\alpha_{\mathrm{h}}}{\alpha_{\mathrm{s}}^{2}} \exp \left[\frac{1}{2}-\frac{0.703}{\alpha_{\mathrm{h}}^{2}}\right] \simeq 0.97 \frac{\alpha_{\mathrm{h}}}{\alpha_{\mathrm{s}}^{2}}$.

For the case shown in Fig. 3, this ratio is $\mathcal{R}=9.7 \times 10^{3}$. We summarize the differences for the total synchrotron fluence SEDs and light curves (numerical values hold for the cases shown in Figs. 2 and 3):

D1) in the high injection case the synchrotron SED peaks at a photon energy, which is a factor $2 x_{B}=1.4 \alpha_{\mathrm{h}}^{2}=1.4 \times 10^{-4}$ less than the peak in the small injection case;

D2) the high injection energy peak value decreases at early times $t<\alpha t_{\mathrm{s}} / 3$ more rapidly than the small injection energy peak value;

D3) the high injection SED is a broken power-law with spectral indices +0.5 below and -0.5 above the peak energy $x_{B} \ll 1$, respectively, and it cuts off exponentially at photon energies $x>1$. Below the peak energy $x_{B}$ the time of maximum synchrotron intensity decreases as $t_{\max } \propto \epsilon^{-1 / 2}$, whereas above the peak energy $x_{B}$ it decreases more rapidly as $t_{\max } \propto \epsilon^{-1 / 2}$ due to the severe additional SST losses;

D4) the small injection SED is a single power-law with spectral index +0.5 below the peak energy $x_{\mathrm{p}}=0.5$, and it cuts off exponentially at photon energies $x>1$. Here, the time of maximum synchrotron intensity decreases as $t_{\max } \propto \epsilon^{-1 / 2}$ at all energies $x<1$ because in the small injection case the SST-losses do not contribute.

All four features are quantitatively visible in Figs. 1-3. These predicted differences for the total synchrotron fluence SED and the synchrotron light curve behaviors provide a conclusive test for high or low injection energy conditions in blazars.

\section{Synchrotron and SSC fluence SEDs and light curves from numerical radiation code}

In Fig. 4 we show the photon energy variation in the light curve peak time calculated with the numerical radiation code of Böttcher et al. (1997) using a magnetic field strength $b=1$ and an injection Lorentz factor $\gamma_{0}=10^{4}$ for the high $\left(\alpha_{\mathrm{h}}=100\right)$, small $\left(\alpha_{\mathrm{s}}=0.1\right)$ and intermediate $(\alpha=1)$ injection cases. The numerical variations for $\alpha_{\mathrm{h}}=100$ and $\alpha_{\mathrm{s}}=0.1$ are in perfect agreement with the different power-law variations found analytically, which are included in Fig. 4 for orientation, and they confirm our earlier findings. For the intermediate case $\alpha=1$, for which no analytical solution is possible, one observes a peak time variation $t_{\mathrm{S}} \propto v^{-1}$ that lies between the $v^{-3 / 2}$-variation and the $v^{-1 / 2}$-variation of the high and small injection cases.

In Figs. 5-7, we show the numerical synchrotron and SSC fluence SEDs using a magnetic field strength $b=1$ and an injection Lorentz factor $\gamma_{0}=10^{4}$ for the high $\left(\alpha_{\mathrm{h}}=100\right)$, small $\left(\alpha_{\mathrm{s}}=0.1\right)$ and intermediate $(\alpha=1)$ injection cases. The first

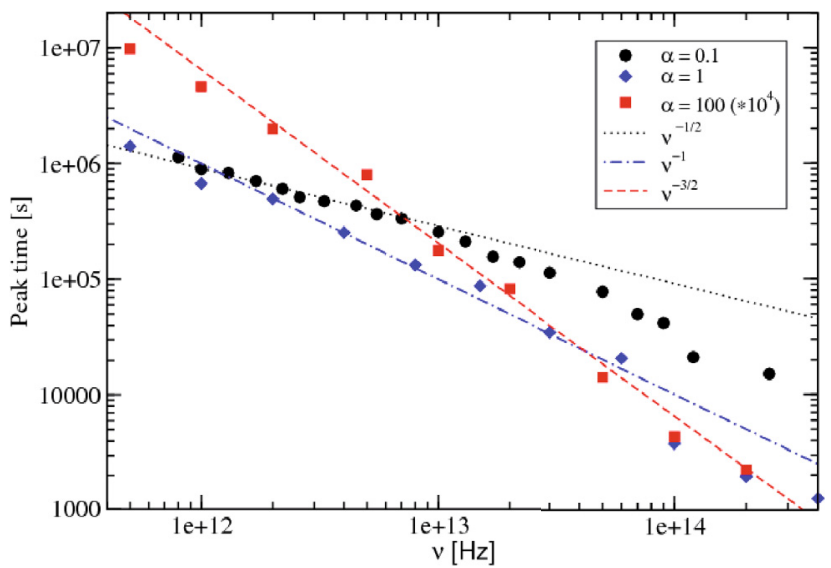

Fig. 4. Numerically calculated synchrotron light curve peak times for small $\left(\alpha_{\mathrm{s}}=0.1\right)$, intermediate $\alpha=1$, and high $\left(\alpha_{\mathrm{h}}=100\right)$ injection conditions calculated for $\gamma_{0}=10^{4}$ and $b=1$.

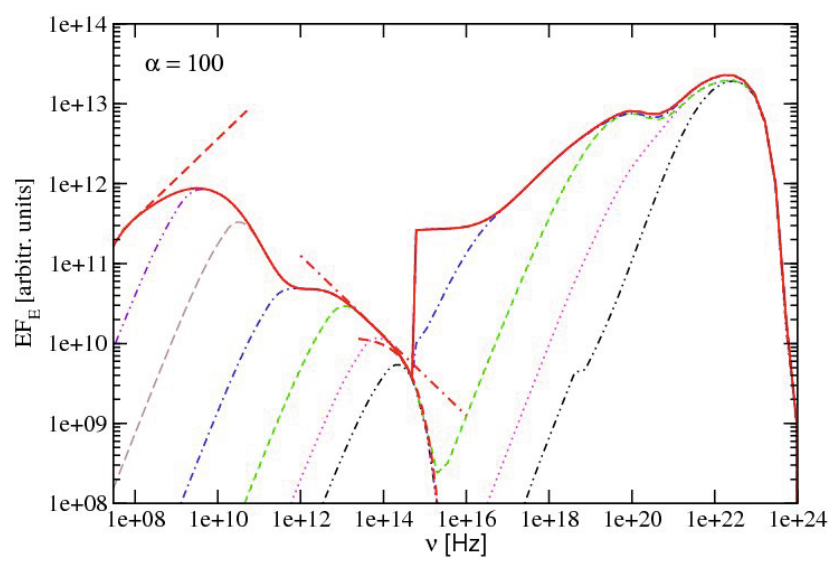

Fig. 5. Numerically calculated fractional and total synchrotron and SSC fluence SEDs for high $\left(\alpha_{\mathrm{h}}=100\right)$ injection conditions calculated for $\gamma_{0}=10^{4}$. The full curves show the total fluence SEDs. The dashed and dot-dashed lines show the asymptotic behavior of the total synchrotron fluence according to Eq. (67). The fractional fluences are shown at approximately logarithmically spaced times. The SSC emission has been artificially cut off at low frequencies as it would otherwise overwhelm the high-energy end of the synchrotron emission.

two synchrotron SEDs are in remarkable agreement with the analytical SEDs shown in Fig. 3. In particular, the numerical SEDs confirm all four predicted differences listed in the last section. For orientation, we have plotted in Figs. 5 and 6 the asymptotic synchrotron spectra (Eqs. (67) and (50)).

The good agreement of the numerically and analytically calculated synchrotron radiation fluence distributions confirms a posteriori the validity of the assumptions made in the analytical calculations, in particular the neglect of higher order SSC losses for the high injection case.

In Fig. 7 we show the numerical synchrotron and SSC fluence SEDs using a magnetic field strength $b=1$ and an injection Lorentz factor $\gamma_{0}=10^{4}$ for the intermediate $(\alpha=1)$ injection case. The spectral form of the synchrotron and SSC fluences are similar to the small injection case shown in Fig. 6, but the SSC peak fluence is only a factor 3 less than the synchrotron peak fluence. Apparently, for no strong influence of nonlinear SST losses occurs for $\alpha=1$. For orientation, we have also plotted the analytic asymptotic behavior of the total synchrotron fluence of the small $\alpha$-case according to Eq. (50) in this plot, which agrees well with the numerical spectral behavior. 


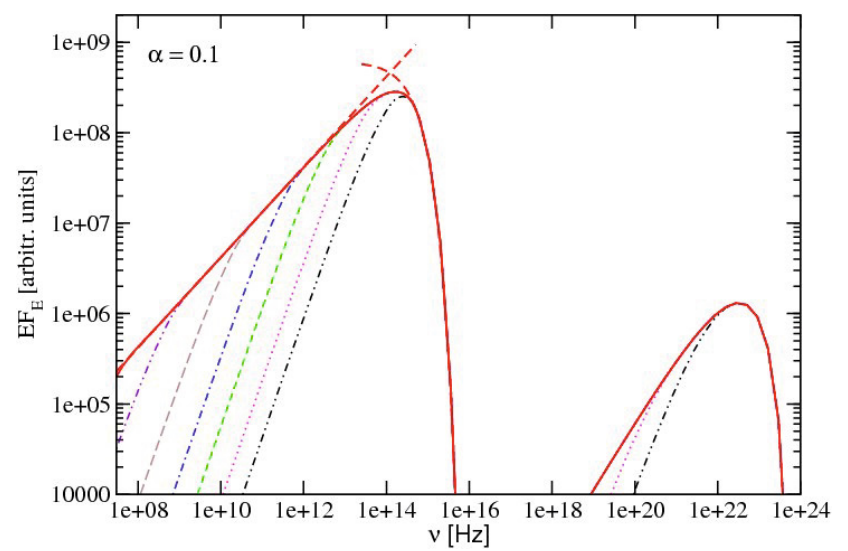

Fig. 6. Numerically calculated fractional and total synchrotron and SSC fluence SEDs for small $\left(\alpha_{\mathrm{s}}=0.1\right)$ injection conditions calculated for $\gamma_{0}=10^{4}$. The full curves show the total fluence SEDs. The dashed lines show the asymptotes according to Eq. (50). The fractional fluences are shown at approximately logarithmically spaced times.

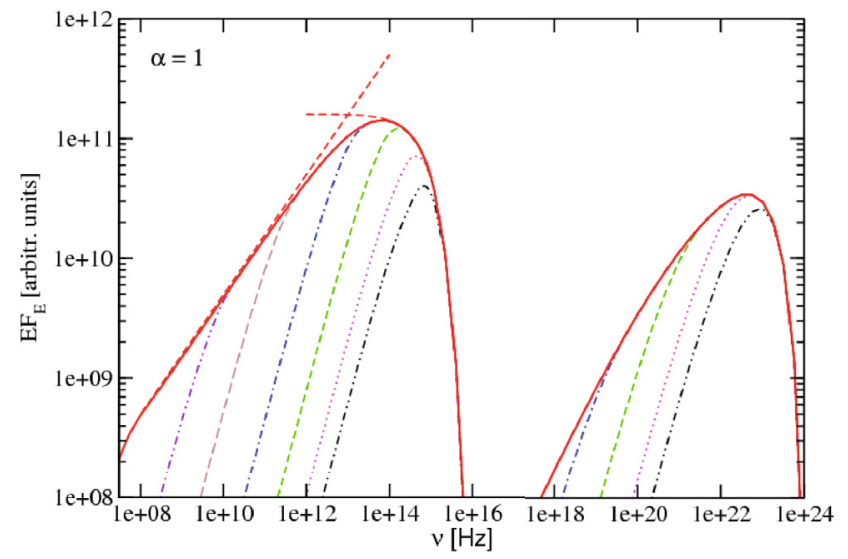

Fig. 7. Numerically calculated fractional and total synchrotron and SSC fluence SEDs for intermediate $(\alpha=1)$ injection conditions calculated for $\gamma_{0}=10^{4}$. The full curves show the total fluence SEDs. The dashed and dot-dashed lines show the asymptotic behavior of the small $\alpha$-case according to Eq. (50). The fractional fluences are shown at approximately logarithmically spaced times.

The radiation code also yields the SSC fluence SEDs. We note from Figs. 5-7 that for the high injection case, the SSC SED has a much higher amplitude than the synchrotron SED, whereas the opposite holds for the low and intermediate injection cases. Moreover, all SSC SEDs peak at the same photon energy, although the SSC peak value in the high injection case is a factor $2 \times 10^{7}$ larger than in the small injection case.

\section{Summary and conclusions}

We have presented an analytical solution to the synchrotron and nonlinear synchrotron-self-Compton cooling in the Thomson regime (SST) of monoenergetic electrons. Based on our analytical solution, we evaluated the time-dependent synchrotron emissivity and time-integrated fluences. We find qualitatively different results depending on whether electron cooling is initially Compton-dominated (high injection energy parameter $\alpha$ ), or it is always synchrotron dominated (low $\alpha$ ). In the low- $\alpha$ case, the resulting fluence spectrum exhibits a simple exponentially cut-off power-law behavior, $S_{v} \propto v^{1 / 2} \mathrm{e}^{-v / v_{0}}$. In contrast, in the high- $\alpha$ case, we find a broken power-law with exponential cutoff, parametrized in the form $S_{v} \propto v^{1 / 2} \frac{v_{B}}{v+v_{B}} \mathrm{e}^{-v / v_{0}}$. The analytically calculated synchrotron fluence SEDs and light curve peak times agree well with the corresponding numerically calculated quantities using the radiation code of Böttcher et al. (1997), which justifies the validity of the assumptions made in the analytical calculations.

Based on our analysis we propose the following interpretation of multiwavelength blazar SEDs. Blazars, where the $\gamma$-ray fluence is much stronger than the synchrotron fluence, are regarded as high injection-energy sources. Here, the synchrotron fluence should exhibit the symmetric broken power-law behavior Eq. (67) around the synchrotron peak energy that is a factor $\left(\alpha_{\mathrm{h}} \gamma_{0}\right)^{2}$ lower than the SSC peak energy. Below and above $v_{B}$, the synchrotron light curve peak times exhibit different frequency dependences $t_{\max }\left(v<v_{B}\right) \propto v^{-1 / 2}$ and $t_{\max }\left(v>v_{b}\right) \propto$ $v^{-3 / 2}$, respectively, resulting from the additional severe SSTlosses at $v>v_{B}$.

Blazars, where the $\gamma$-ray fluence is much less than the synchrotron fluence, are regarded as small injection-energy sources. Here, the synchrotron fluence exhibits the single power-law behavior (D4) up to a higher synchrotron peak energy that is a factor $\gamma_{0}^{2}$ lower than the SSC peak energy. In this case, the synchrotron light curve peak time exhibits the standard linear synchrotron cooling decrease $t_{\max }(v) \propto v^{-1 / 2}$ at all frequencies.

If the injection Lorentz factor $\gamma_{0}$ and the size of the source are the same, different values of the injection parameter $\alpha$ result from different total numbers of instantaneously injected electrons, see Eq. (19); for example, the high injection case $\alpha_{\mathrm{h}}=100$ results for $N_{50}=4.7$, whereas the low injection case $\alpha_{\mathrm{s}}=0.1$ needs $N_{50}=4.7 \times 10^{-6}$.

Acknowledgements. We thank the referee for his/her constructive comments. R.S. acknowledges support from the German Ministry for Education and Research (BMBF) through Verbundforschung Astroteilchenphysik grant 05A08PC1 and the Deutsche Forschungsgemeinschaft through grants Schl 201/20-1 and Schl 201/23-1. M.B. acknowledges support from NASA through Fermi Guest Investigator grant NNX09AT82G and Astrophysics Theory Program Grant NNX10AC79G. This work was completed when M.B. was visiting guest scientist of the Research Department Plasmas with Complex Interactions at Ruhr-University Bochum.

\section{Appendix A: Determination of electron distribution functions for low and high injection energies}

\section{A.1. Low injection energy $\gamma_{0} \ll \gamma_{B}$}

In the case of low injection energies $\gamma_{0} \ll \gamma_{B}$, the injection parameter $\alpha \ll 1$ is less than unity, so that the argument of the arctan-function in Eq. (15) is always greater than unity. For all values of $T$ and $y$, Eq. (15) then simplifies to

$x_{0}+T(y) \simeq K_{0} y+C_{0}$,

and with $C_{0}=x_{0}$ to

$T(y) \simeq K_{0} y$.

In terms of $y$, the solution (12) then reads with $x_{0}>0$

$S\left(x, x_{0}, y\right)=q_{0} H\left[x-x_{0}\right] \delta\left(x-x_{0}-K_{0} y\right)$,

yielding

$$
\begin{aligned}
n\left(\gamma, \gamma_{0}, t\right) & =\frac{q_{0}}{\gamma^{2}} H\left[\gamma_{0}-\gamma\right] \delta\left(\gamma^{-1}-\gamma_{0}^{-1}-D_{0} t\right) \\
& =q_{0} H\left[\gamma_{0}-\gamma\right] \delta\left(\gamma-\frac{\gamma_{0}}{1+D_{0} \gamma_{0} t}\right)
\end{aligned}
$$

which agrees with the standard linear synchrotron cooling solution. 


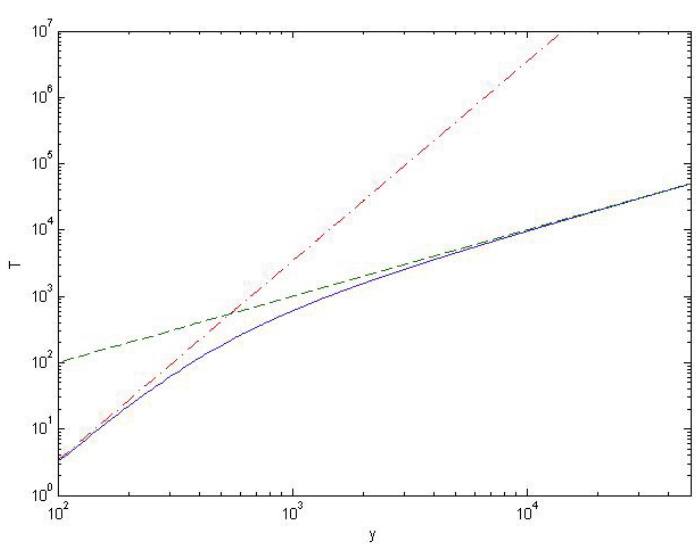

Fig. A.1. Comparison of exact solution $y(T)$ (full curve) with asymptotic solution $y_{1}(T)$ at early times (dashed curve) and asymptotic solution $y_{2}(T)$ at late times (dotted curve) for the high injection energy case with $q_{5}=1, x_{0}=10^{-4}$ and $K_{0}=1$, corresponding to $\alpha=3 \times 10^{6}$.

\section{A.2. High injection energy $\gamma_{0} \gg \gamma_{B}$}

In the case of high injection energies $\gamma_{0} \gg \gamma_{B}$ the injection parameter $\alpha \gg 1$ is greater than unity. With the injection parameter (17) we rewrite Eq. (15) as

$K_{0} y+C_{1}=\alpha x_{0}\left[\frac{1+\frac{T}{x_{0}}}{\alpha}-\arctan \left(\frac{1+\frac{T}{x_{0}}}{\alpha}\right)\right]$

For times $0 \leq T \leq T_{\mathrm{c}}$, where

$T_{\mathrm{c}}=(\alpha-1) x_{0}$,

we expand $\arctan (x) \simeq x-\left(x^{3} / 3\right)$ to third order in $x$ to obtain

$K_{0} y_{1}+C_{1} \simeq \frac{x_{0}}{3 \alpha^{2}}\left(1+\frac{T}{x_{0}}\right)^{3}$,

or

$y_{1}=\frac{\left(x_{0}+T\right)^{3}}{3 q_{0}}-C_{2}$.

With $T=0$ for $y=0$, the integration constant $C_{2}=x_{0}^{3} / 3 q_{0}$ is fixed so that

$y_{1}=\frac{\left(x_{0}+T\right)^{3}}{3 q_{0}}-\frac{x_{0}^{3}}{3 q_{0}}$.

This solution is valid for $T \leq T_{\mathrm{c}}$, corresponding with Eq. (A.6) to

$0 \leq y \leq y_{\mathrm{c}}=\frac{x_{0}^{3}}{3 q_{0}}\left(\alpha^{3}-1\right)=\frac{x_{0}}{3 \alpha^{2} K_{0}}\left(\alpha^{3}-1\right)$.

For times $T \geq T_{\mathrm{c}}$ or $y \geq y_{\mathrm{c}}$, the argument of the arctan-function in Eq. (A.5) is greater than unity, yielding

$K_{0} y_{2}+C_{3} \simeq x_{0}+T$,

or the linear relation

$y_{2}=\frac{x_{0}+T}{K_{0}}-C_{4}$.
The constant $C_{4}$ is determined by the equality of the two solutions $y_{1}\left(T_{\mathrm{c}}\right)=y_{2}\left(T_{\mathrm{c}}\right)=y_{\mathrm{c}}$ at $T_{\mathrm{c}}$ providing

$C_{4}=\frac{\alpha x_{0}}{K_{0}}+\frac{x_{0}^{3}}{3 q_{0}}\left(1-\alpha^{3}\right)=\frac{x_{0}^{3}}{3 q_{0}}\left[1+2 \alpha^{3}\right]$,

so that

$y_{2}=\frac{x_{0}+T}{K_{0}}-\frac{2 q_{0}^{1 / 2}}{3 K_{0}^{3 / 2}}-\frac{x_{0}^{3}}{3 q_{0}}$.

In Fig. A.1 we compare the two approximate solutions (A.9) and (A.14) with the exact solution (16). The agreement is reasonably good.

Both approximate solutions (A.9) and (A.14) can be inverted to yield

$$
\begin{aligned}
T_{1}\left(y<y_{\mathrm{c}}\right) & =\left[3 q_{0} y+x_{0}^{3}\right]^{1 / 3}-x_{0} \\
& =x_{0}\left[\left(1+\frac{3 \alpha^{2} K_{0} y}{x_{0}}\right)^{1 / 3}-1\right]
\end{aligned}
$$

and

$T_{2}\left(y \geq y_{\mathrm{c}}\right)=x_{0}\left[\frac{1}{3 \alpha^{2}}\left(\frac{3 \alpha^{2} K_{0} y}{x_{0}}+1+2 \alpha^{3}\right)-1\right]$.

In terms of $y$ we obtain from Eq. (11) the two solutions

$S_{1}\left(x, x_{0}, y<y_{\mathrm{c}}\right)=$

$q_{0} H\left[x-x_{0}\right] H\left[y_{\mathrm{c}}-y\right] \delta\left(x-x_{0}\left(1+\frac{3 \alpha^{2} K_{0} y}{x_{0}}\right)^{1 / 3}\right)$

and

$$
\begin{aligned}
S_{2}\left(x, x_{0}, y>y_{\mathrm{c}}\right) & =q_{0} H\left[x-\alpha x_{0}\right] H\left[y-y_{\mathrm{c}}\right] \delta \\
& \times\left(x-\left[K_{0} y+\frac{1+2 \alpha^{3}}{3 \alpha^{2}} x_{0}\right]\right) .
\end{aligned}
$$

We then find for early times

$n_{1}\left(\gamma, \gamma_{0}, t<t_{\mathrm{c}}\right)=$

$$
\begin{array}{r}
q_{0} H\left[\gamma_{0}-\gamma\right] H\left[t_{\mathrm{c}}-t\right] \delta\left(\gamma-\frac{\gamma_{0}}{\left(1+3 \alpha^{2} \gamma_{0} K_{0} A_{0} t\right)^{1 / 3}}\right)= \\
q_{0} H\left[\gamma_{0}-\gamma\right] H\left[t_{\mathrm{c}}-t\right] \delta\left(\gamma-\frac{\gamma_{0}}{\left(1+3 q_{0} \gamma_{0}^{3} A_{0} t\right)^{1 / 3}}\right)
\end{array}
$$

which agrees with the nonlinear SST solution of S, his Eq. (28). At late times,

$n_{2}\left(\gamma, \gamma_{0}, t \geq t_{\mathrm{c}}\right)=$

$q_{0} H\left[\gamma_{B}-\gamma\right] H\left[t-t_{\mathrm{c}}\right] \delta\left(\gamma-\frac{\gamma_{B}}{\frac{1+2 \alpha^{3}}{3 \alpha^{3}}+\gamma_{B} K_{0} A_{0} t}\right)$,

which is a modified linear cooling solution. Both solutions show that, at time

$$
\begin{aligned}
t_{\mathrm{c}}=\frac{y_{\mathrm{c}}}{A_{0}} & =\frac{\alpha^{3}-1}{3 \alpha^{3} \gamma_{B} D_{0}} \simeq \frac{1}{3 \gamma_{B} D_{0}} \\
& =\frac{2.6 \times 10^{8}}{\gamma_{B} b^{2}} \mathrm{~s}=\frac{1.2 \times 10^{6} N_{50}^{1 / 2}}{R_{15} b^{2}} \mathrm{~s},
\end{aligned}
$$

the electrons have cooled to the characteristic Lorentz factor $\gamma_{B}$.

Page 10 of 11 


\section{Appendix B: Calculation of synchrotron radiation constants}

Here we determine the constants

$c(k) \equiv \int_{0}^{\infty} \mathrm{d} x x^{k} C S(x)$

for general values of $k>-1 / 3$. The integral (B.1) is directly related to the integral $G^{\prime}(s)$ computed in Eq. (29) of Crusius \& Schlickeiser (1986) if $s=2 k+1$. This yields

$$
\begin{aligned}
c(k)= & \frac{2}{\pi} G^{\prime}(2 k+1)=\frac{2^{k}(3 k+5)(3 k-1)}{18 \pi^{1 / 2} k(k+2)} \\
& \times \frac{\Gamma\left[\frac{k+1}{2}\right] \Gamma\left[\frac{3 k+1}{6}\right] \Gamma\left[\frac{3 k-1}{6}\right]}{\Gamma\left[\frac{k}{2}\right]}
\end{aligned}
$$

with the triplication formula of gamma functions for $z=(3 k-$ 1)/6, Eq. (B.2) simplifies to

$c(k)=\frac{2^{k} \pi^{1 / 2}(3 k+5)(3 k-1)}{3^{\frac{3 k+2}{2}} k(k+2)} \frac{\Gamma\left[\frac{3 k-1}{2}\right]}{\Gamma\left[\frac{k}{2}\right]}$.

We obtain $c(1 / 2)=0.95302$ and $c(3 / 2)=0.67015$.

\section{References}

Abdo, A. A., Ackermann, M., Ajello, M., et al. 2009, ApJ, 699, 817 Abdo, A. A., Ackermann, M., Agudo, I., et al. 2010a, ApJ, 716, 30

Abdo, A. A., Ackermann, M., Ajello, M., et al. 2010b, Nature, 463, 919

Aharonian, F. A., Akhperjanian, A. G., Bazer-Bachi, A. R., et al. (HESS

Collaboration) 2005, A\&A, 442, 895

Aharonian, F. A., Akhperjanian, A. G., Bazer-Bachi, A. R., et al. (HESS Collaboration) 2007, A\&A, 470, 475

Bloom, S. D., \& Marscher, A. P. 1996, ApJ, 461, 657

Böttcher, M. 2007, Astroph. \& Space Sci. 309, 95

Böttcher, M., \& Bloom, S. D. 2000, AJ, 119, 469

Böttcher, M., Mause, H., Schlickeiser, R. 1997, A\&A, 324, 395

Crusius, A., \& Schlickeiser, R. 1986, A\&A, 164, L16

Crusius, A., \& Schlickeiser, R. 1988, A\&A, 196, 327

Dermer, C. D., \& Schlickeiser, R. 2002, ApJ, 575, 667

Fossati, G., et al. 2008, ApJ, 667, 906

Hinton, J. A., \& Hofmann, W. 2009, ARA\&A, 47, 523

Kardashev, N. S. 1962, Sov. Astron. J. 6, 317

Madejski, G., Sikora, M., Jaffe, T., et al. 1999, ApJ, 521, 145

Maraschi, L., Celotti, A., \& Ghisellini, G. 1992, ApJ, 397, L5

Sambruna, R. M., Urry, C. M., Maraschi, L., et al. 1997, ApJ, 474, 639

Schlickeiser, R. 2009, MNRAS, 398, 1483 (S)

Schlickeiser, R., \& Lerche, I. 2007, A\&A, 476, 1 